Auf der Kaiserl. Sternwarte in Strassburg, mitgetheilt von Prof. E. Becker.

Kurz vor der Zeit des Eintritts bedeckte sich der Himmel im Osten und erst um die Zeit der grössten Phase wurde die Sonne durch die Wolken hindurch sichtbar. Beobachtungen erst ganz kurz vor dem Austritt möglich. Austritt nur durch Wolken beobachtet, die gerade im Augenblick der Berührung wieder die Sonne ganz verdecken.

Grosser Refractor

Fernrohr von Merz auf der Plattform des Refractorbaues

$\begin{array}{lc}\text { Oeffn. } & \text { Vergr. } \\ 487 \mathrm{~mm} & 305 \\ 102 & 70\end{array}$

Beob. Kobold Tetens
Austritt M. Z. Str. $18^{\mathrm{h}} 7^{\mathrm{m}} 33^{\mathrm{s}}$ 18736

\title{
Ueber die Sonnenfinsterniss vom 7. Juni 1899 und deren photographische Aufnahme.
}

Der Eintritt des Mondes wurde nicht beobachtet. Den Austritt beobachtete Frau $G$. Wolf um $18^{\mathrm{h}} \times 3^{\mathrm{m}} 19^{\mathrm{s}} 6$, ich selbst um $18^{\mathrm{h}} 13^{\mathrm{m}} 26^{\mathrm{s}} .0$ M. Z. Königstuhl. Den Austritt der Mitte eines hohen Mondberges sah ich um $18^{\mathrm{h}} 8^{\mathrm{m}} 4{ }^{\mathrm{s}} \cdot 5$. Die Ränder waren recht scharf trotz des niederen Standes. Frau Wolf beobachtete am 3 zöll. Merz, ich selbst am 6 zöll. Reinfelder.

Ich habe diesmal etwas, was ich schon ofters vergeblich versucht hatte, wieder versucht, nämlich Serienaufnahmen der Finsterniss auf derselben Platte vorzunehmen; und zwar dieses Mal mit Erfolg.

Es handelt sich darum, so wie ich es seit Jahren bei Mondfinsternissen ausgefürt habe, eine Reihe von Bildern neben einander auf dieselbe Platte zu machen. Das Instrument bleibt ruhig stehen, während von Zeit zu Zeit in genau bestimmbarem Moment der Deckel des Objectivs geöffnet wird. Dann erhält man aus der gegenseitigen Lage und Distanz der Bilder die Constanten für die Vermessung der Platte.

Beim Mond ging dies sehr gut, weil eine Belichtung von I Secunde, wie ich sie dabei verwenden konnte, scharfe Bilder mit der engsten Blende des 6 zöll. Voigtländer-Dublets giebt und die Platte durch die ganze Reihe der Aufnahmen nicht verschleiert wird. Bei der Sonne versagte das Ver- fahren wegen der zu grossen Helligkeit. Denn es konnte von einem rapiden Momentverschluss dieser Grösse (für die ganze Reihe der Aufnahmen) keine Rede sein und ohne einen solchen wurde die Platte schon von einer Aufnahme bereits schwarz, geschweige denn von 8 oder mehr.

Wenn man aber, wie ich es jetzt gethan habe, Diapositivplatten benutzt und einen Entwickler anwendet, wie ich ihn unten beschreibe, bekommt man schöne Resultate. Ich habe 8 Sonnenaufnahmen auf derselben Platte; die Bilder sind dabei haarscharf, richtig belichtet und die Platte ist glasklar geblieben.

Von einem kurz vorher bereits kräftig benutzten Rodinalentwickler löste ich soviel in Wasser auf, dass das Lösungsverhältniss des Rodinals zu Wasser I : 10000 betrug und fügte auf 100 dieser Lösung $10 \mathrm{ccm}$ einer 10 procentigen Bromkalilösung hinzu; ich stellte also nichts anderes her als einen äusserst schwachen und harten Standentwickler. Nach einer Stunde war die Entwickelung beendet und die Bilder vorziuglich gelungen. Es war dabei ziemlich gleichgültig, ob die Bilder $1 \frac{1}{2}$, oder $1 / 4$ Secunde belichtet waren. Die Sonnenflecken sind auf allen sichtbar und die Hörner der Sonne wegen der Feinkörnigkeit der Diapositivplatten von ungewohnter Schärfe.

Heidelberg, den I 0 . Juni 1899 .

Max Wolf.

\section{Observations de planètes et de la comète $1899 \mathrm{I}$}

faites à l'Equatorial de ro pouces de l'Observatoire de Genève par F. Pidoux astronome.

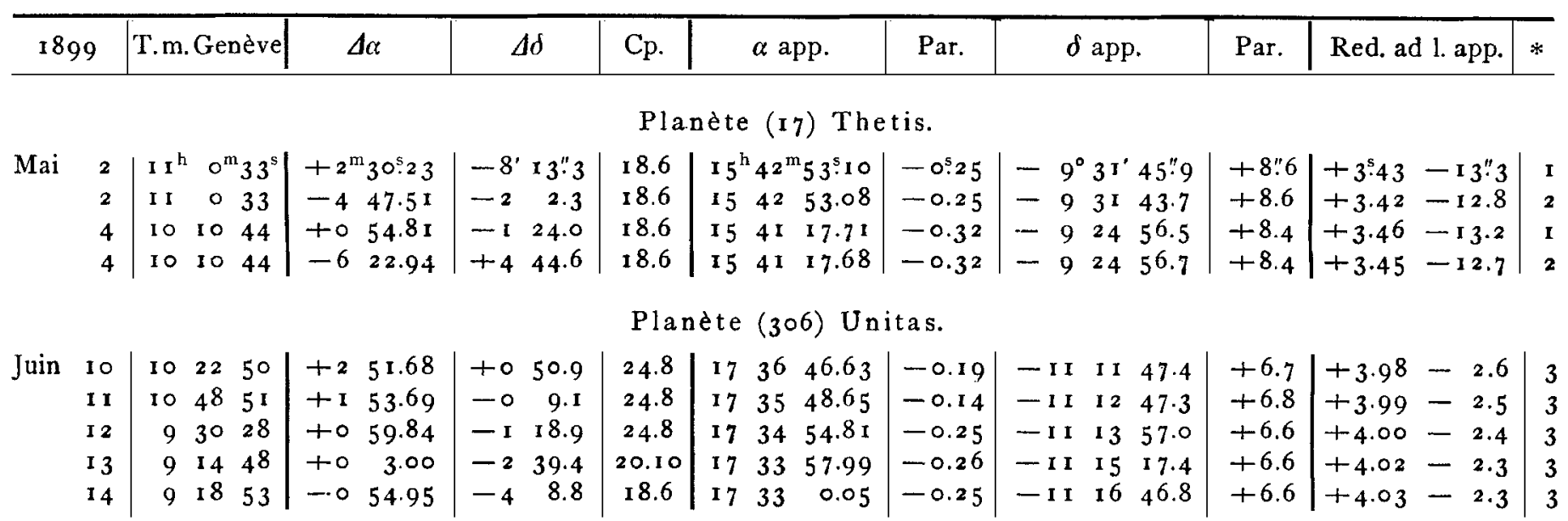




\begin{tabular}{l|l|l|l|l|l|l|l|l|l|l|}
\hline 1899 & T.m.Genève & $\Delta \alpha$ & $\Delta \delta$ & Cp. & $\alpha$ app. & $\log p . \Delta$ & $\delta$ app. & $\log p . \Delta \mid$ Red. ad l. app. & $*$ \\
\hline
\end{tabular}

Comète 1899 I (a, Swift).

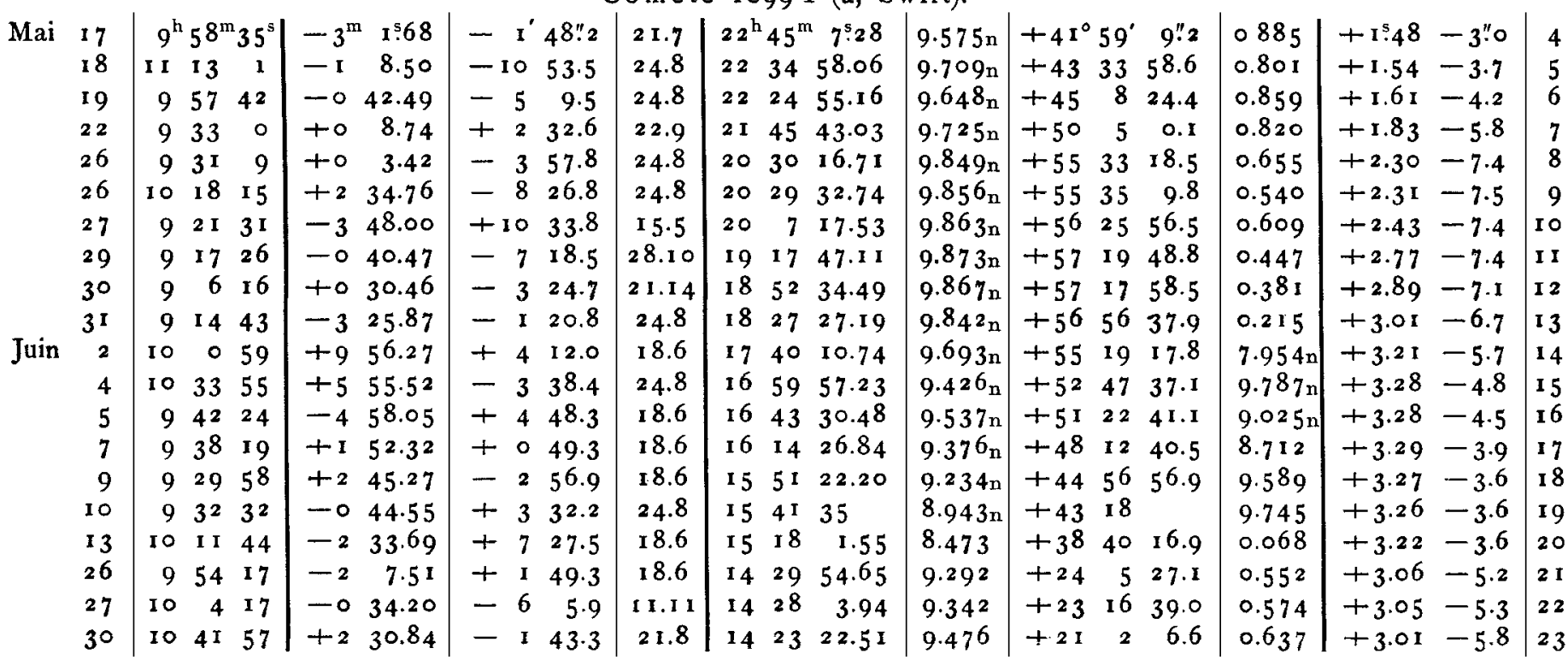

Positions moyennes des étoiles de comparaison.

\begin{tabular}{|c|c|c|c|c|c|c|c|}
\hline * & $\alpha 1899.0$ & $\delta$ I 899.0 & Autorité & * & $\alpha \mathrm{I} 899.0$ & $\delta_{1} 899.0$ & Autorité \\
\hline I & $15^{\mathrm{h}} 40^{\mathrm{m}} \times 9^{\mathrm{s}} \cdot 44$ & $-9^{\circ} 23^{\prime} \times 9^{\prime \prime} 3$ & AG. Ottak. Z. $4_{4}$ r & I I & $19^{\mathrm{h}} \mathrm{r} 8^{\mathrm{m}} 24^{\mathrm{s}} 8 \mathrm{I}$ & $+57^{\circ} 27^{\prime} \times 4^{\prime \prime} 7$ & AG. Hels. 10399 \\
\hline 2 & $1547 \quad 37.17$ & -92928.6 & AG. Ottak. Z. I 4 I & I 2 & $18 \quad 52 \quad$ I.1 4 & +572130.3 & AG. \\
\hline 3 & $1733 \quad 50.97$ & $\begin{array}{lll}-11 & 12 & 35.7\end{array}$ & Cord. GC. 23934 & I 3 & I8 $30 \quad 50.05$ & $+56 \quad 58 \quad 5.4$ & $45 \mathrm{~d}$ Drac. $\mathrm{AC}$ \\
\hline 4 & $22 \quad 48 \quad 7.48$ & +42 I 0.4 & AG. Bonn I 7 I9I & 14 & 173011.26 & +551511.5 & $\nu^{1}$ Drac. Fond.C. 24 \\
\hline 5 & $22 \quad 3^{6} \quad 5.02$ & +434455.8 & I Lacertae. AG. Bonn & $\begin{array}{l}15 \\
36\end{array}$ & $\begin{array}{lll}16 & 53 & 58.43 \\
16 & 48 & 25.27\end{array}$ & $\begin{array}{l}+52 \\
+51\end{array}$ & $\begin{array}{l}\text { AG. Cambr. } 5 \text { II } 9 \\
A G \text { Cambr. } 5002\end{array}$ \\
\hline 6 & $\begin{array}{lll}22 & 25 & 36.04\end{array}$ & +45 I3 38.1 & AG. Bonn I 6792 & I 7 & 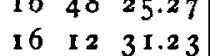 & $\begin{array}{r}+511757 \cdot 3 \\
+481155 \cdot 1\end{array}$ & $\begin{array}{l}\text { AG. Cambr. } 5092 \\
\text { AG. Bonn } 10437\end{array}$ \\
\hline 7 & $21 \quad 45 \quad 32.46$ & $+50 \quad 233.3$ & Milieu de la double: AG. & 18 & I5 54833.66 & +445957.4 & AG. Bonn 1 \\
\hline & & & $\begin{array}{l}\text { Bonn I6017-18, AG. } \\
\text { Cambr. } 7292-93\end{array}$ & $\begin{array}{l}19 \\
20\end{array}$ & $\begin{array}{lll}15 & 42 & 16 \\
15 & 20 & 32.02\end{array}$ & $\begin{array}{l}+43 \text { I } 5 \\
+383^{2} 53.0\end{array}$ & $\begin{array}{l}\text { BD. }+43^{\circ} .2525 \\
\text { AG.Lund Z.6,26,28,29,69I }\end{array}$ \\
\hline 8 & $20 \quad 30 \quad 10.99$ & +553723.7 & AG. Hels. 11465 & 2 I & 143159.10 & +24343.0 & AG. Berlin B. 5112 \\
\hline 9 & $20 \quad 26 \quad 55.67$ & +554344.1 & AG. Hels. I I 4 I I & 22 & $14 \quad 28 \quad 35.09$ & +232250.2 & AG. Berlin B. 5098 \\
\hline 10 & 20 II 3.10 & $+5^{6}$ & 33 Cygne. Fd.C. 500 & 23 & 1420048.66 & +213 & AG. Berlin B. $5 \circ 7 \mathrm{I}$ \\
\hline
\end{tabular}

Comparaison des observations avec les éphémérides $(\mathrm{O}-\mathrm{C})$.

\begin{tabular}{l|l|l}
\hline 1899 & $\Delta a$ & $\Delta \delta$ \\
\hline
\end{tabular}

Planète (I 7) Thetis.

[B. J. 1901$]$.

\begin{tabular}{ll|l|l} 
Mai & 2 & +7.55 & -13.7 \\
& 2 & +7.53 & -11.5 \\
4 & +7.57 & -12.5 \\
& 4 & +7.54 & -12.7
\end{tabular}

Remarques aux observations de la comète $1899 \mathrm{I}$.

Mai ı. La comète n'a pu être observée plus tôt; $\mid$ plus faible que l'étoile double de comparaison $8^{\mathrm{m}} \cdot 2$ et $8^{\mathrm{m}} \cdot 5$. elle était masquée par le bâtiment principal. - Mai 22. - Mai 27. La comète passe devant une très petite étoile Observation génée par le clair de lune. La comète est bien qui se trouve près du centre du noyau, à droite en haut.

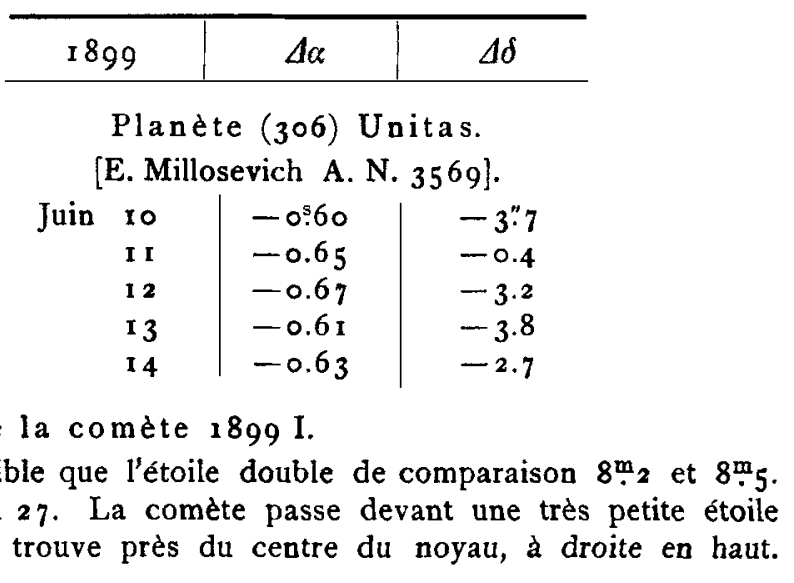


- Juin 4. La comète est plus ronde et l'éventail s'est développé sur toute la périphérie quoique toujours moins prononcé à droite. Le noyau est plus lumineux, plus étoffé et le centre constitue un point très brillant. On peut le pointer comme une étoile mais il ne scintille pas. - Juin 5. Le ciel se couvre peu à peu et l'étoile de comparaison n'est plus visible dans le chercheur; en revanche, le noyau de la comète

Genève, $I^{\text {er }}$ juillet 1899 . persiste et semble se dégager lumineux et phosphorescent sur le fond sombre du ciel. On peut encore pointer sur le centre du noyau comme sur une étoile. - Juin 9. L'éclat de la comète a beaucoup diminue; toutefois, le centre se dégage encore comme un point brillant sur le reste du noyau. - Juin 30. Comete à la limite de visibilité pour des mesures micrométriques de quelque précision.

\section{Posizione dell' Osservatorio di Catania ed anomalie di gravità.}

Le osservazioni meridiane di stelle per la longitudine furono fatte nell' estate 1894 per cinque notti, osservando io in Catania ed il Prof. Zona in Palermo e per cinque notti, osservando io in Palermo ed il Prof. Zona in Catania. Gli strumenti adoperati erano del tipo Bamberg, a cannocchiale spezzato.

In ogni sera si osservava un gruppo di 13 stelle, poi si trasmettevano i segnali telegrafici per la longitudine, quindi si osservava un altro gruppo di 13 stelle.

Il Prof. Saija ha fatto intanto questo calcolo preliminare, adottando $\mathrm{i}$ valori medii degli elementi variabili in ciascuna notte d'osservazione. Si farà dopo il calcolo rigoroso, il quale potrà dare un risultato leggermente diverso. La media ponderata delle ro differenze di longitudine fra l' Osservatorio di Catania e l' Osservatorio di Palermo è

$$
6^{\mathrm{m}} 54^{\mathrm{s}}: 8 \text {. }
$$

Quindi la longitudine dell' Osservatorio di Catania è

$$
\begin{aligned}
& 1^{\circ} 4^{\prime} 27^{\prime \prime}=0^{\mathrm{h}} 6^{\mathrm{m}} 45^{\mathrm{s}} \cdot 8 \text { Est Berlino } \\
& 23^{6} \text { I } 8=0 \text { ro } 25.2 \text { Est Roma (C. R.) } \\
& \text { I } 4456=0 \quad 5059.7 \text { Est Parigi } \\
& \text { I } 559=\text { I } \circ 20.6 \text { Est Greenwich } \\
& 2 \text { 1 } 129=\text { I } 259.9 \text { Est San Fernando }
\end{aligned}
$$

La latitudine di Catania (strumento dei passaggi), determinata dal Prof. Zona nel 1894 , è $+37^{\circ} 30^{\prime}$ I $3^{\prime \prime} 25$.

\begin{tabular}{|c|c|c|}
\hline & Latitudine $\mathrm{N}$ & Long. W di Castanea \\
\hline & $37^{\circ} 30^{\prime}$ I $3{ }^{\prime \prime} 25$ & $0^{\circ} 26^{\prime} \quad 8^{\prime \prime} 79$ \\
\hline & $37 \quad 30 \quad 11.39$ & $0 \quad 2631.75$ \\
\hline Differenza $A-G$ & +1.86 & +22.96 \\
\hline
\end{tabular}

Confrontando queste coordinate astronomiche colle geodetiche, ottenute dall' Istituto Geografico Militare con provenienza da Castanea (prov. di Messina), si ha:

Osservatorio di Catania, 24 Giugno 1899.

\section{F. Pidoux.}

Perciò la deviazione ortodromica locale in longitudine è

$$
22.96 \cos \varphi=+18.2
$$

e la deviazione risultante della verticale è

1 8:"3 nell' azimut $84^{\circ}$ ro'.

Dunque l'Etna che ha la cima a $28 \mathrm{~km}$ a NNW di Catania non produce deviazione del filo a piombo; ciò si accorda col fatto trovato da me col pendolo Sterneck, che nell' Etna vi è un grande difetto di massa rispetto a $\mathrm{Ca}$ tania, corrispondente ad uno strato di altezza circa $1 / 3$ del cono vulcanico.

L' intensità relativa della gravità in Catania, da me determinata coll' apparato Sterneck in Ottobre 1897 e Marzo 1898 , in media è

$$
9.80075 \text {; }
$$

ridotta al livello del mare risulta:

$$
9.80085
$$

mentre il valore teorico, dato dalla formola d'Helmert, è

$$
9 \cdot 79925 \text {. }
$$

Quindi l' anomalia dell' intensità di gravità in Catania è : +0 m.00160.

A. Riccò.

Comet 1899 I (a, Swift). Corr. der Ephem. (A. N. 3574 ): Juli $3^{1}+9^{\mathrm{s}} 3+0^{\prime} 6$. Sehr schwach, Kern I $4^{\mathrm{m}}$. V. Cerulli.

Personalnotiz. The Superintendent of the U. S. Coast and Geodetic Survey has designated Dr. Frank Schlesinger, Columbia University, New York City, to take charge of the variation of latitude observations at Ukiah, California, in accordance with the plans of the International Geodetic Association.

Notiz. Die Abonnenten, welche die Zeitschrift auf dem Wege des Buchhandels beziehen, können die aus 4 Bogen bestehende Nr. 3584-85 erst nach einiger Zeit erhalten, da die gesammte, für die Buchhandlung W. Mauke Söhne in Hamburg bestimmte Auflage durch einen Unfall auf der Post beschädigt worden ist und neu gedruckt werden muss.

Inhalt zu Nr. 3586. T.F. F. See. The Determination of the Potential of a heterogeneous Sphere upon itself, with an extension of Helmholtz's Theory of the Heat of the Sun. 177. - Ph. Fauth. Besondere Wahrnehmungen an dem Planeten Mars 1899. 181. Beobachtungen der partiellen Sonnenfinsterniss 1899 Juni 7. 183. - M. Wolf. Ueber die Sonnenfinsterniss vom 7. Juni I899 und deren photographische Aufnahme. 187. - F. Pidoux. Observations de planètes et de la comète 1899 1. 187. - A. Riccò. Posizione dell' Osservatorio di Catania ed anomalie di gravità. I9I. - V. Centlli. Comet I899 I (a, Swift). I9I. - Personalnotiz. I9I. - Notiz. I9I. 